\title{
Kinematics and binaries in young stellar aggregates ${ }^{\star \star \star \star}$ II. NGC $6913 \equiv$ M 29
}

\author{
C. Boeche ${ }^{1}$, U. Munari ${ }^{1}$, L. Tomasella ${ }^{1}$, and R. Barbon ${ }^{2}$ \\ 1 Osservatorio Astronomico di Padova, Sede di Asiago, 36012 Asiago (VI), Italy \\ 2 Osservatorio Astrofisico del Dipartimento di Astronomia, Universitá di Padova, 36012 Asiago (VI), Italy
}

Received 10 July 2003 / Accepted 7 October 2003

\begin{abstract}
Between 1996 and 2003 we obtained 226 high resolution spectra of 16 stars in the field of the young open cluster NGC 6913, to constrain its main properties and study its internal kinematics. Twelve of the program stars turned out to be members, one of them probably unbound. Nine are binaries (one eclipsing and another double lined) and for seven of them the observations allowed us to derive the orbital elements. All but two of the nine discovered binaries are cluster members. In spite of the young age (a few Myr), the cluster already shows signs that could be interpreted as evidence of dynamical relaxation and mass segregation. However, they may be also the result of an unconventional formation scenario. The dynamical (virial) mass as estimated from the radial velocity dispersion is larger than the cluster luminous mass, which may be explained by a combination of the optically thick interstellar cloud that occults part of the cluster, the unbound state or undetected very wide binary orbit of some of the members that inflate the velocity dispersion and a high inclination for the axis of possible cluster angular momentum. All the discovered binaries are hard enough to survive average close encounters within the cluster and do not yet show signs of relaxation of the orbital elements to values typical of field binaries.
\end{abstract}

Key words. stars: binaries: spectroscopic - stars: early type - ISM: bubbles - Galaxy: open clusters and associations: general - Galaxy: open clusters and associations: individual: NGC 6913

\section{Introduction}

This is the second paper of a series devoted to the results of a long term, high resolution spectroscopic study of early type members of young open clusters, trapezium systems and OB associations. The aims of this series are discussed in Paper I (Munari \& Tomasella 1999).

NGC 6913, the topic of this paper, is a young open cluster harboring O-type members and lying close to the plane of the Galaxy $\left(\alpha=20^{\mathrm{h}} 23^{\mathrm{m}} \cdot 9, \delta=+38^{\circ} 32^{\prime}(\mathrm{J} 2000) ; l=76^{\circ} .92\right.$, $b=+0.61$ ). Despite appearing in the Messier catalog as M 29, few papers in the literature deal with it, furthermore they show some disagreement in the results. Cluster distance is reported to be $2.2 \mathrm{kpc}$ by Morgan \& Harris (1956) and Massey et al. (1995), $1.5 \mathrm{kpc}$ by Joshi et al. (1983), and $1.1 \mathrm{kpc}$ by Hoag et al. (1961), while Tifft (1958) suggested that NGC 6913 is indeed the result of two separate groups of stars, one at $1.6 \mathrm{kpc}$ and the other somewhere between 1.9 and $2.4 \mathrm{kpc}$.

Send offprint requests to: $\mathrm{U}$. Munari,

e-mail: munari@pd.astro.it

$\star$ Table 2 is only available in electronic form at http://www . edpsciences.org

$\star \star$ Table 4 is only available in electronic form at the CDS via anonymous ftp to cdsarc.u-strasbg.fr $(130.79 .128 .5)$ or via http://cdsweb.u-strasbg.fr/cgi-bin/qcat?]/A+A/415/145
The mean and differential reddening span a range of values too: $\left\langle E_{B-V}\right\rangle=0.78, \Delta E_{B-V}=0.64$ according to Joshi et al. (1983), $\left\langle E_{B-V}>=0.71\right.$ and $\Delta E_{B-V}=1.82$ for Wang $\& \mathrm{Hu}$ (2000), and $\left\langle E_{B-V}\right\rangle=1.03$ following Massey et al. (1995). Similarly, estimated ages span from 0.3-1.75 Myr of Joshi et al. (1983) to 10 Myr of Lyngå (1987).

The internal and galactic kinematics of NGC 6913 have not so far been investigated in the literature. The cluster radial velocity used by Hron (1987) in modeling the rotation curve of the Galaxy, $-25 \mathrm{~km} \mathrm{~s}^{-1}$, was assembled from scanty literature data that apparently missed all the brightest cluster members, and is far from our much more accurate and representative $-16.9( \pm 0.6) \mathrm{km} \mathrm{s}^{-1}$ value (see Sect. 3.2). The internal kinematics and binary content of NGC 6913 are unknown because no detailed radial velocity study of its members has been pursued, and proper motions investigations (Sanders 1973; Dias et al. 2002) are not deep and accurate enough for a firm membership segregation over a wide range of magnitudes, do not cover all candidate members and do not allow resolution of the internal kinematics.

In this paper we aim to look in more detail at NGC 6913 general properties (like astrometric membership, photometry, reddening, distance, mass and age) and to present and discuss the results of our extensive spectroscopic study of NGC 6913 based on 226 high resolution spectra monitoring of 16 stars in 
Table 1. Program stars. The first four columns give our identification number (cf. finding chart in Fig. 1), and that assigned by Hoag et al. (1961), Sanders (1973) and Kazlauskas \& Jasevicius (1986). $V$ and $B-V$ are Tycho-2 $V_{\mathrm{T}}$ and $(B-V)_{\mathrm{T}}$ transformed into Johnson system following Bessell (2000) prescriptions. $U-B$ is the median of the measurements by Massey et al. (1995), Joshi et al. (1983) and Hoag et al. (1961). Star \#10 is reported as a short period variable by Peña et al. (2001).

\begin{tabular}{rrrrccccll}
\hline \hline$\#$ & H61 & S73 & KJ86 & HD & HIP/TYC & $V$ & $B-V$ & $U-B$ & notes \\
\hline 1 & 1 & 135 & 125 & 194378 & HIP 100586 & 8.603 & 0.431 & +0.07 & V2031 Cyg \\
2 & 2 & 159 & 145 & 229239 & HIP 100612 & 9.035 & 0.730 & -0.14 & \\
3 & 3 & 157 & 144 & 229238 & TYC 3152 1325 1 & 8.935 & 0.801 & -0.07 & \\
4 & 4 & 149 & 138 & 229234 & TYC 3152 1369 1 & 8.979 & 0.638 & -0.20 & \\
5 & 5 & 125 & 118 & 229221 & TYC 3152 1451 1 & 9.260 & 0.767 & -0.25 & V1322 Cyg \\
6 & 6 & 139 & 127 & 229227 & HIP 100600 & 9.419 & 0.632 & -0.18 & \\
7 & 7 & 174 & 156 & 229253 & TYC 3152 236 1 & 10.171 & 0.099 & -0.31 & \\
8 & 8 & 147 & 136 & & TYC 3152 1309 1 & 10.388 & 0.733 & -0.17 & \\
9 & 9 & 146 & 134 & 229233 & TYC 3152 1137 1 & 10.494 & 0.346 & +0.02 & \\
10 & 10 & 182 & 162 & 229261 & TYC 3152 1415 1 & 10.510 & 0.252 & -0.31 & var \\
11 & 11 & 178 & 158 & & TYC 3152 1019 1 & 11.307 & 0.518 & +0.26 & \\
12 & 12 & 122 & 115 & & TYC 3152 676 1 12.091 & 0.025 & -0.06 & \\
13 & 13 & 167 & 150 & & TYC 3152 1467 1 & 11.692 & 0.862 & +0.83 & \\
14 & 14 & 148 & 137 & & TYC 3152 1423 1 & 11.552 & 0.426 & +0.04 & \\
15 & & 143 & 132 & & TYC 3152 54 1 & 11.534 & 1.120 & +0.28 & \\
16 & & & 103 & & TYC 3152 1453 1 & 10.983 & 0.528 & -0.30 & \\
\hline
\end{tabular}

Table 2. Journal of observations. $D$ is the dispersions $(\AA /$ pix $)$ at $\mathrm{H} \alpha$ ( 0.19 corresponding to unbinned spectra, 0.38 to $2 \times$ binned spectra), and $\Delta \lambda$ is the wavelength coverage. The last column gives the program stars observed in each given run (the table is only available in electronic form).

the field of the cluster over the time span 1996-2003. These observations are used to constrain the internal velocity dispersion, the cluster galactic motion, the individual rotational velocities, and the internal kinematical and evolutionary status of the cluster. Spectroscopic orbits are calculated for the discovered binary stars.

\section{Spectroscopic observations}

Table 1 summarizes the main properties of the 16 selected program stars, and Fig. 1 provides a finding chart for them. The program stars have been spectroscopically observed over the period 1996-2003 with the $1.82 \mathrm{~cm}$ telescope and Echelle+CCD spectrograph of the Astronomical Observatory of Padova at Asiago (Italy). Table 2 provides the journal of observations. The instrumental set-up, spectra extraction and calibration, accuracies, etc. are identical to Paper I and the reader is referred there for details.

\subsection{Spectral classification and radial velocities}

Scanty information exists on the spectral classification of the program stars. Wang \& $\mathrm{Hu}$ (2000) derived spectral types from low resolution spectra $(5.3 \AA /$ pix $)$ covering the range 4200-6900 A. Kazlauskas \& Jasevicius (1986) obtained photoelectric photometry in the Vilnius system, that we have converted into spectral types using the reddening-free color parameters $Q$ defined by Strayžis (1977) appropriate for the $R_{V}=A_{V} / E_{B-V}=3.6$ reddening law that applies to NGC 6913

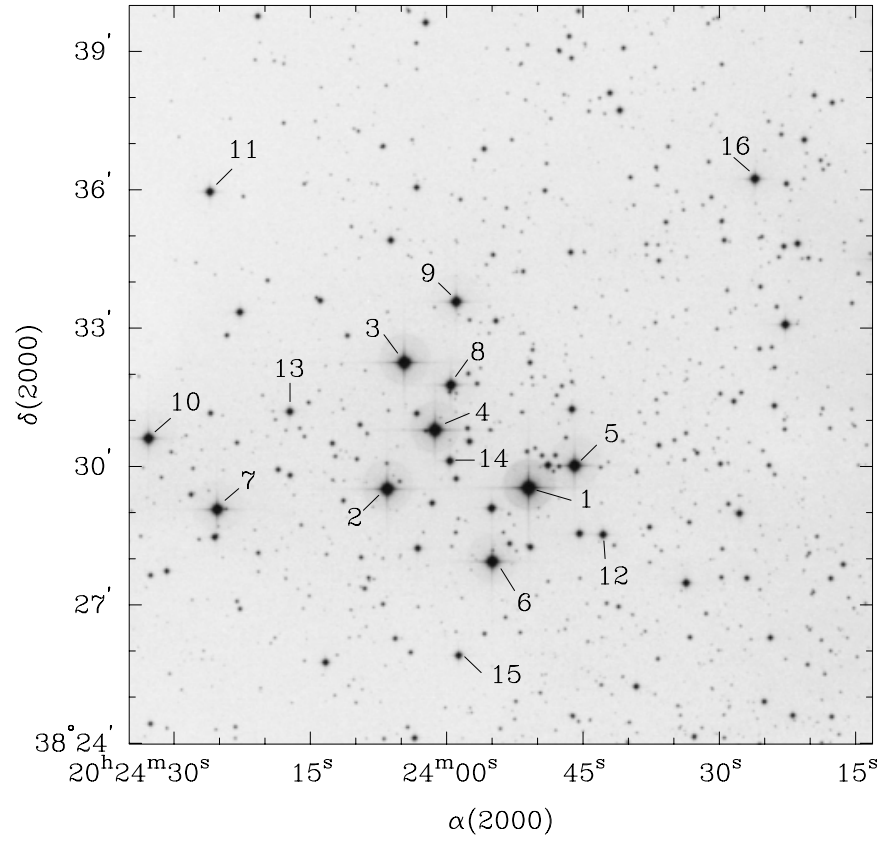

Fig. 1. Finding chart for NGC 6913 program stars.

according to Johnson (1962). We have also derived spectral classification of the program stars using our Echelle spectra, classified against the Yamashita et al. (1977) spectral atlas. Even if spectral classification of Echelle spectra has to be carried out with care (lines to be compared normally fall in different Echelle orders), nevertheless the resulting spectral types look quite reasonable, and, given the far superior spectral resolution and high $\mathrm{S} / \mathrm{N}$, also are possibly more accurate than those of Wang \& Hu (2000). The three estimates of the spectral type are compared in Table 3 . The last two columns of the table give the reddening and distance when Fitzgerald (1970) intrinsic colors and our spectral classification are compared to $V, B-V$ 
Table 3. Spectral types of the program stars from Wang \& $\mathrm{Hu}$ (2000), from photometry in the Vilnius system by Kazlauskas \& Jasevicius (1986) transformed by us into spectral types following Strayžis (1977), and from classification of our Echelle spectra against the Yamashita et al. (1977) reference spectral atlas. The last columns give the $E_{B-V}$ (from Fitzgerald 1970 intrinsic colors) and the spectrophotometric distances for our spectral classification and the photometry in Table 1.

\begin{tabular}{rlcccc}
\hline \hline & \multicolumn{3}{c}{ spectral type } & & \\
\cline { 2 - 4 }$\#$ & WH00 & Vilnius & our & $E_{B-V}$ & $d(\mathrm{kpc})$ \\
1 & F0 III & F1 V & F0 III & 0.11 & 0.2 \\
2 & B0 I & B1 Ia & B0 I & 0.97 & 2.0 \\
3 & B0 I & B1 Ia & B0 I/II & 1.06 & 1.4 \\
4 & O7 II & B1 Ia & O9 Ib & 0.92 & 2.3 \\
5 & B0 IIIe & & B0 IIIe & 1.07 & 1.2 \\
6 & B0 II & B2 Ia & B0 V & 0.93 & 1.1 \\
7 & B4 II & B3 V & B5 IV & 0.26 & 1.6 \\
8 & B0 III & B0 III & B0 Ib/II & 0.99 & 3.0 \\
9 & A4 V & F0 V & A4 V & 0.23 & 0.3 \\
10 & B6 II & B2 V & B5 V/IV & 0.41 & 1.2 \\
11 & & A5 V & A5 III/II & 0.39 & 1.9 \\
12 & & B2 V & B2 V & 0.26 & 5.3 \\
13 & G6 III & & G5 III & 0.00 & 1.1 \\
14 & A8 II & A4 V & F7 IV & 0.00 & 0.7 \\
15 & B1 II & B3 Ib & B1 III & 1.38 & 1.6 \\
16 & & B1 II & B0 V & 0.83 & 2.8 \\
\hline
\end{tabular}

photometry in Table 1 . The positions of the program stars on the reddening-corrected HR diagram are shown in Fig. 2.

Radial velocities from individual observations (hereafter referred to as epoch radial velocities) of the program stars are given in Table 4. For $\mathrm{O}$ and $\mathrm{B}$ type program stars they are based on individual measurement of He I and He II lines. For the other, cooler program stars the radial velocities come from measurement of the metallic absorptions lines (mainly Fe I, Mg I, Ti II). The radial velocities of the Be program star \#5 pertain to the emission lines, which completely fill the helium and hydrogen absorption lines.

\subsection{Binaries and orbital solutions}

About half of the program stars have turned out to be spectroscopic binaries. Table 5 summarizes the barycentric velocity, the membership and the binary status based on epoch radial velocities in Table 4. Table 6 gives the spectroscopic orbits computed for all the binary stars but \#6 and 16, which are clearly binaries but the available radial velocities are not enough to determine the orbital period and thus to allow to derive an orbital solution. Therefore the $R V_{\odot}$ quoted for stars \#6 and 16 in Table 5 is the mean of the measurements, not the barycentric velocity, and the two velocities tend to differ with increasing eccentricity and paucity of measurements. Consequently, the $R V_{\odot}$ of stars \#6 and 16 quoted in Table 5 which differ by slightly more than $3 \sigma$ from the cluster mean velocity cannot

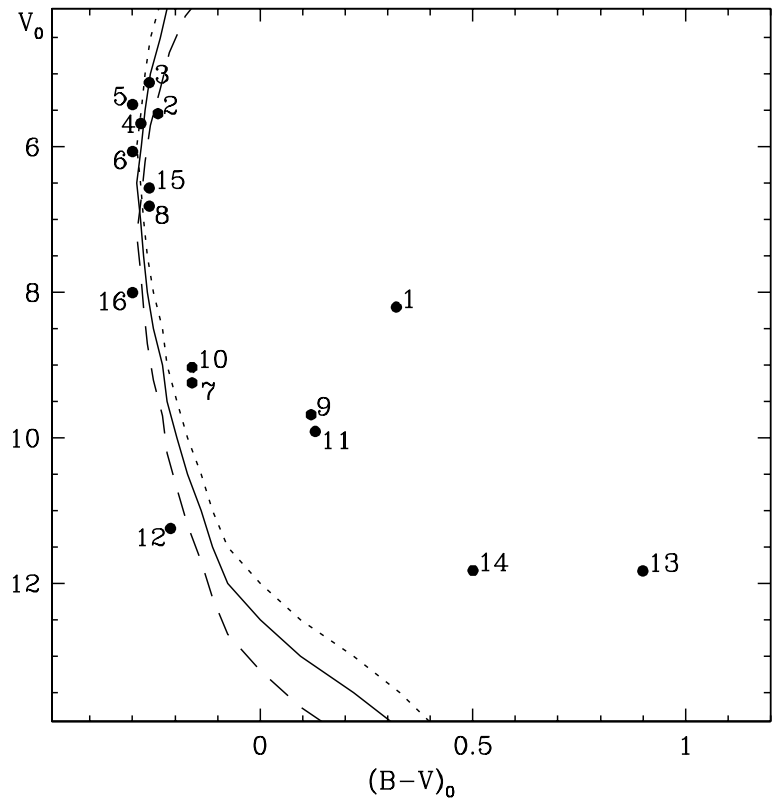

Fig. 2. The program stars on the reddening corrected $V_{0},(B-V)_{0}$ diagram using $E_{B-V}$ from Table 3 and $V, B-V$ photometry from Table 1 for $R_{V}=A_{V} / E_{B-V}=3.6$ appropriate for NGC 6913 according to Johnson (1962). The isochrone for solar metallicity and $5 \mathrm{Myr}$ is from Bertelli et al. (1994) and it is scaled to $m-M=10.5$ (dotted line), $m-M=11.0$ (solid line) and $m-M=11.7$ (dashed line). It is evident how the cluster distance cannot be well constrained. In this paper we adopt a $1.6 \mathrm{kpc}$ distance.

Table 4. Example of the table containing the epoch radial velocities (and their errors) for the program stars, available in full only in electronic form at the CDS.

\begin{tabular}{crr}
\hline \hline & & \\
HJD & $R V_{\odot}$ & err \\
50263.424 & -19.8 & 1.5 \\
50296.514 & -19.5 & 1.2 \\
50299.487 & -18.1 & 0.9 \\
50316.566 & -18.8 & 0.9 \\
50324.479 & -19.3 & 0.9 \\
50333.434 & -19.8 & 0.9 \\
50333.559 & -19.8 & 0.9 \\
50655.500 & -20.5 & 0.9 \\
50671.566 & -19.9 & 1.7 \\
51895.302 & -26.3 & 0.9 \\
52781.402 & -18.9 & 1.0 \\
\hline
\end{tabular}

be considered as a firm indication that stars \#6 and 16 are field stars.

Similarly to Paper I, the spectroscopic orbits have been obtained with a Fortran code written by Roger F. Griffin (Cambridge University) and adapted to run under GNU/Linux by us.

Program stars \#1-7 have been observed also by Liu et al. $(1989,1991)$ who reported some epoch radial velocities for them. Such data appear affected by large errors for the $\mathrm{O}$ and B stars (program stars \#2-7), which make them useless in our analysis. They are instead in good agreement with our velocities for star \#1, which is much cooler having a spectral 
Table 5. Heliocentric radial velocity (with its standard error) of the program stars, binary status and cluster membership according to radial velocities, and projected rotational velocity (with its standard error). The radial velocity of the binary stars is the barycentric velocity from the orbital solutions in Table 6 . The last column gives the projected rotation period $P_{\text {rot }} / \sin i$ of the solved binaries to be compared with the orbital period.

\begin{tabular}{rccccc}
\hline \hline$\#$ & $\begin{array}{c}R V_{\odot} \\
\left(\mathrm{km} \mathrm{s}^{-1}\right)\end{array}$ & binary & member & $\begin{array}{c}V_{\text {rot }} \sin i \\
\left(\mathrm{~km} \mathrm{~s}^{-1}\right)\end{array}$ & $\begin{array}{c}P_{\text {rot }} / \sin i \\
(\text { days })\end{array}$ \\
\hline 1 & $+8.6 \pm 0.5$ & yes & no & $26 \pm 1$ & 5.0 \\
2 & $-17 \pm 6$ & yes & yes & $128 \pm 3$ & 10 \\
3 & $-18.5 \pm 0.6$ & yes & yes & $84 \pm 2$ & 15 \\
4 & $-15.0 \pm 0.6$ & yes & yes & $103 \pm 2$ & 10 \\
5 & $-19.4 \pm 0.3$ & no & yes & & \\
6 & $-25.6 \pm 0.9$ & yes & (yes) & $253 \pm 5$ & \\
7 & $-18 \pm 3$ & yes & yes & $46 \pm 5$ & 3.8 \\
8 & $-15.5 \pm 0.4$ & no & yes & $67 \pm 2$ & \\
9 & $-5.6 \pm 0.5$ & no & no & $22 \pm 2$ & \\
10 & $-14.8 \pm 0.7$ & no & yes & $64 \pm 7$ & \\
11 & $0 \pm 3$ & yes & no & $41 \pm 6$ & 11 \\
12 & $-17 \pm 6$ & yes & yes & $165 \pm 7$ & 1.5 \\
13 & $-14.6 \pm 0.2$ & no & yes & $0 \pm 2$ & \\
14 & $-19.9 \pm 0.4$ & no & yes & $11 \pm 3$ & \\
15 & $-16 \pm 2$ & no & yes & $115 \pm 4$ & \\
16 & $-27 \pm 4$ & yes & (yes) & $325 \pm 20$ & \\
\hline
\end{tabular}

type F0 III. The reason for the poor quality of the Liu et al. radial velocities for hot stars probably lies in the shortness of the wavelength range they observed $(\sim 150 \AA)$ and in the fact that it is dominated by $\mathrm{H} \delta$, which we ignored in our analysis given the Balmer progression and its excessive scatter compared to the much more performing $\mathrm{He}$ I and He II lines. It is also worth noticing that Liu et al. did not recognized star \#7 as double lined, in spite of having observed it at orbital phase 0.66 when the velocity separation between the components is $\sim 140 \mathrm{~km} \mathrm{~s}^{-1}$ (cf. Fig. 3) and therefore outstanding.

The spectroscopic orbits of stars \#2 and 11 in Fig. 3 and Table 6 are to be considered quite preliminary, given their small amplitude, high eccentricity and limited number of observations. Further observations are obviously encouraged for these two stars. Photometric observations of the double lined star \#7 are in progress to constrain the orbital inclination and derive individual masses, and they will reported elsewhere when completed.

\subsection{Rotation velocities}

Rotation velocities for the program stars are given in Table 5. They have been derived from He I lines for stars \#2, 3, 4, 6, 7, $8,10,12,15,16$ and $\mathrm{Fe}$ I lines for the remaining ones, following the numerical relations for the Asiago Echelle spectrograph calibrated in Paper I (its Fig. 7). No rotation velocity is derived for the Be program star \#5 because all He I lines are badly affected by emissions. The correspondence of the rotational velocity scale between He I and Fe I lines (which we have been forced to used in all program stars with a spectral type later than B) has been carefully checked on a grid of Kurucz rotationally broadened spectra we have calculated for this purpose.

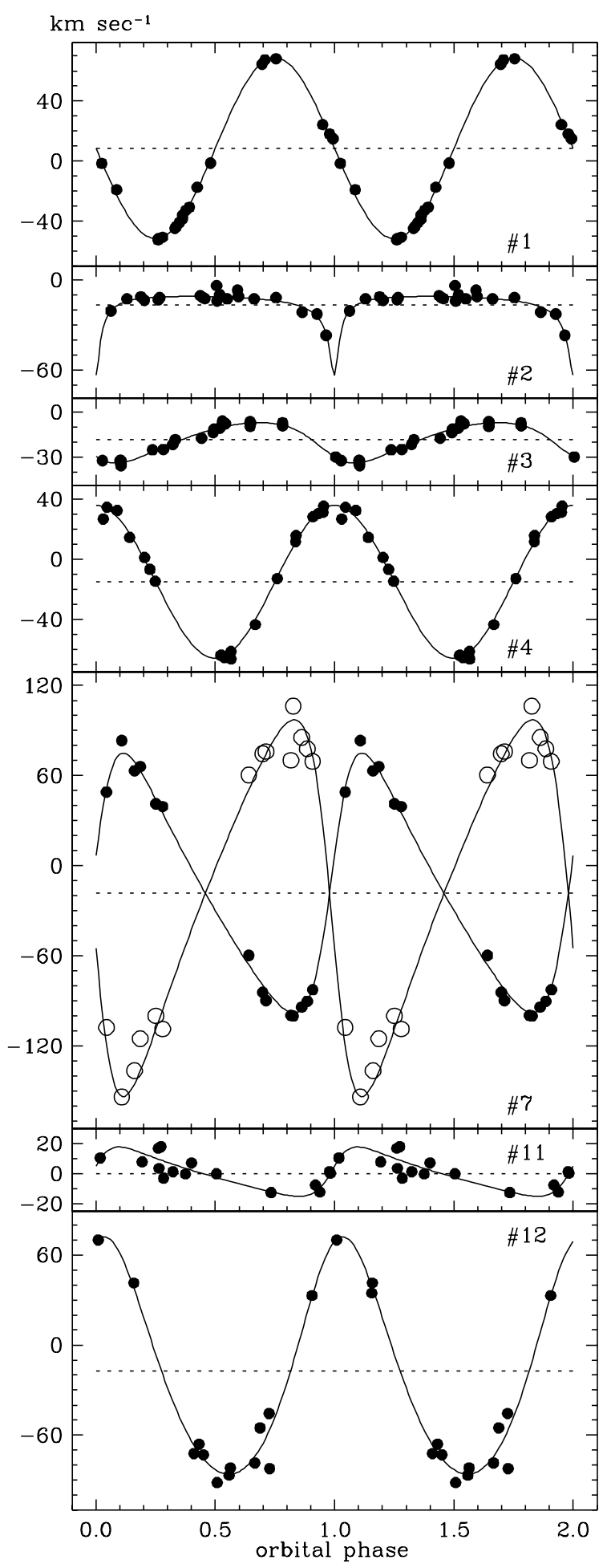

Fig. 3. Orbital solutions for the binary program stars (cf. Table 6).

From the spectral classification in Table 3, the stellar radii over the HR diagram as tabulated by Straižys \& Kuriliene (1981) and the observed $V_{\text {rot }} \sin i$ projected rotation velocity, we present in the last column of Table 5 the projected rotation period $\left(P_{\mathrm{rot}} / \sin i\right)$ for the binaries with an orbital solution in Table 6 . The projected rotation period is obviously an upper limit to the true rotation period. Compared to the orbital period 
Table 6. Orbital solution for the binary stars we discovered in NGC 6913. For binaries \#6 and 16 the epoch radial velocities in Table 4 did not allowed a determination of the orbital period and therefore the derivation of the orbit. The errors are given in parenthesis in units of the last digit. The last raw gives the rms deviation of the solution from the observed radial velocities.

\begin{tabular}{|c|c|c|c|c|c|c|c|c|}
\hline \multirow{2}{*}{\multicolumn{2}{|c|}{ orb. element }} & \multicolumn{7}{|c|}{ program star \# } \\
\hline & & 1 & 2 & 3 & 4 & 7 & 11 & 12 \\
\hline$P$ & (days) & $2.70466(1)$ & $1.7075(1)$ & $697(7)$ & $3.51042(5)$ & $3.4588(1)$ & $1.56842(4)$ & $4.0350(5)$ \\
\hline$e$ & & 0.0 & $0.8(7)$ & $0.21(5)$ & 0.0 & $0.35(2)$ & $0.4(2)$ & $0.13(9)$ \\
\hline$K$ & $\left(\mathrm{~km} \mathrm{~s}^{-1}\right)$ & $60.1(7)$ & $26(6)$ & $13.5(6)$ & $50.9(8)$ & $87(2), 125(5)$ & $16(5)$ & $79(6)$ \\
\hline$\gamma$ & $\left(\mathrm{km} \mathrm{s}^{-1}\right)$ & $+8.6(5)$ & $-17(6)$ & $-18.5(6)$ & $-15.0(6)$ & $-18(3)$ & $0(3)$ & $-17(6)$ \\
\hline$T_{0}$ & $(+2450000)$ & $1132.626(4)$ & $1362.2(1)$ & $652(25)$ & $892.40(1)$ & $923.96(6)$ & $782.3(1)$ & $668.7(3)$ \\
\hline$\omega$ & $(\operatorname{deg})$ & 0.0 & $187(14)$ & $134(12)$ & 0.0 & $282(7)$ & $283(49)$ & $344(26)$ \\
\hline$a \sin i$ & $\left(10^{6} \mathrm{~km}\right)$ & $2.24(2)$ & $0.4(17)$ & $126(6)$ & $2.45(4)$ & $3.84(9)$ & $0.3(1)$ & $4.3(3)$ \\
\hline$f(\mathrm{~m})$ & & $0.061(2)$ & $0.001(9)$ & $0.16(2)$ & $0.048(2)$ & $0.19(1)$ & $0.0005(5)$ & $0.20(4)$ \\
\hline $\mathrm{rms}$ & $\left(\mathrm{km} \mathrm{s}^{-1}\right)$ & 1.41 & 1.09 & 1.36 & 1.72 & 1.57 & 1.49 & 1.57 \\
\hline
\end{tabular}

in Table 6, it can be used to infer the co-rotation status of the binaries.

Star \#1 is an SB1 eclipsing binary and therefore the $\sin i$ projection factor converges toward unity, which allows a direct comparison between rotation and orbital periods, the former being twice the latter. The lack of synchronisation could be related to the primary evolving away from the main sequence and the time scales of the two processes.

Given the masses estimated from the spectral type and the amplitude of radial velocity variation, star \#7 probably has a high inclination too, possibly itself being eclipsing. The rotational velocity in Table 5 pertains to the B5 IV primary, the measurement of the secondary being too uncertain given the difference in brightness. The rotation and orbital periods are quite close, and in view of the uncertainties at play, the primary in star \#7 looks synchronized.

Stars \#3 and 12 are evidently not co-rotating, because the projected rotation period is at least several times shorter than the orbital period in Table 6, and working on $\sin i$ can only enlarge the difference. For the remaining binary stars \#2, 4 and 11, no conclusion can be drawn about the co-rotation status, the projected rotation period being longer than the orbital one.

\subsection{Cluster membership}

Sanders (1973, hereafter S73) has published an astrometric investigation of 228 stars in the field of NGC 6913, identifying 92 possible members. He has however used only one plate pair, with an epoch separation of just $22 \mathrm{yr}$, with moreover the first epoch plate "severely blackened by the moon". Consequently, noting the too large fraction of detected members among the measured stars, he warned that the cluster separation from the field is not satisfactory, and that the member/non-member status he assigned may be frequently wrong. The S73 limiting magnitude is $V=13.8$, with a completness limit not fainter than $V=13.0$ that corresponds to $1.2 M_{\odot}$ on the main sequence of NGC 6913. Dias et al. (2002, hereafter D02) have used proper motions from the Tycho- 2 catalog to accomplish the astrometric member segregation, following the analytical approach of Sanders (1971). They have 24 stars in common with S73. As Fig. 4 shows, for 6 of the 24 common stars the

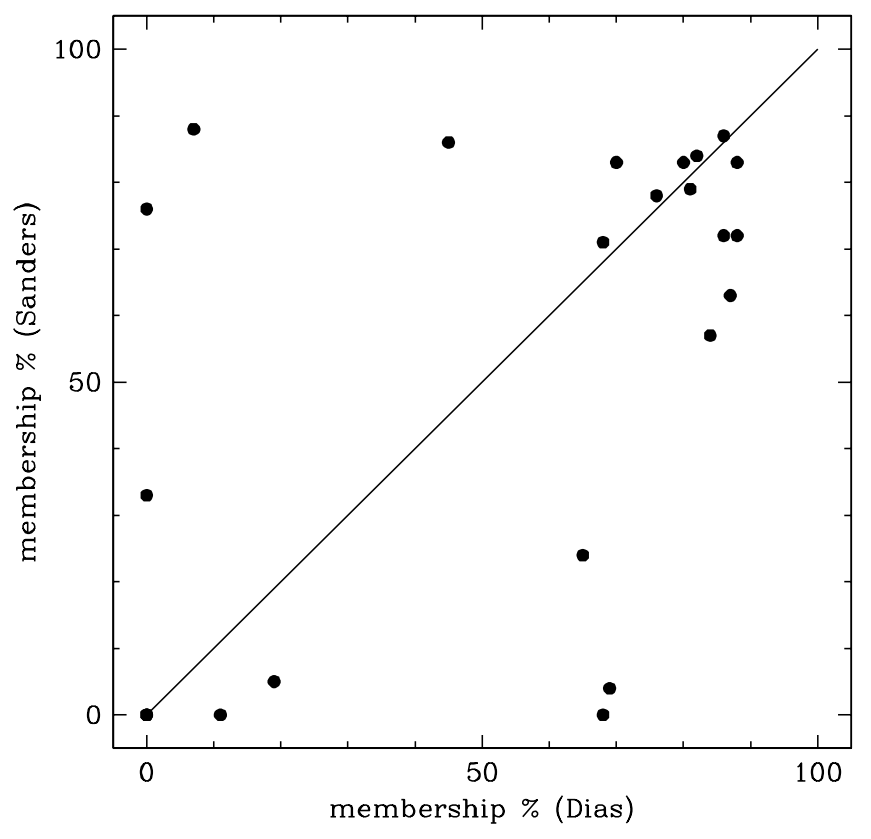

Fig. 4. Comparison between Dias et al. (2002) and Sanders (1973) membership data for the 24 stars in common.

membership status of S73 and D02 is in disagreement, and for the remaining 18 there is a fair agreement.

A firm membership segregation is required for any kinematical investigation of the cluster, and to achieve the best possible result, the astrometric data should be complemented by radial velocities and placing of the program stars on the HR diagram.

The radial velocity distribution of the program stars is presented in Fig. 5, where the cluster grouping at $-16.9 \mathrm{~km} \mathrm{~s}^{-1}$ is evident, with a dispersion of $\sigma=2.0 \mathrm{~km} \mathrm{~s}^{-1}$.

Table 7 summarizes the membership status according to the astrometric investigations of S73 and D02, the photometry presented and discussed by Crawford et al. (1977) and Joshi et al. (1983), the spectral classification of Wang \& Hu (2000), our reddening-free HR diagram of Fig. 2 and the radial velocities in Table 5. Their combination provides our final, adopted membership reported in the last column of Table 7. 


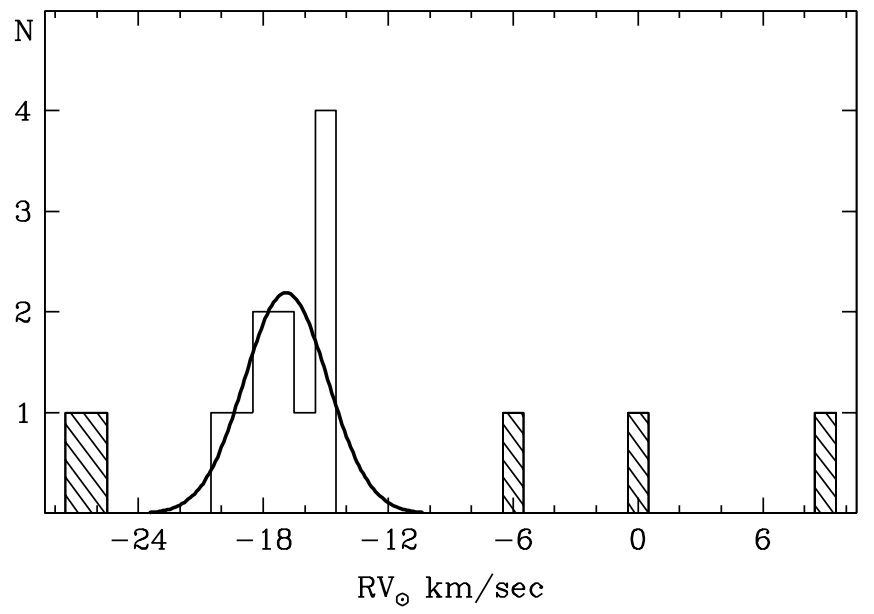

Fig. 5. Radial velocity distribution of the program stars. A Gaussian with center at $R V_{\odot}=-16.9 \mathrm{~km} \mathrm{~s}^{-1}$ and $\sigma=2.0 \mathrm{~km} \mathrm{~s}^{-1}$ fits the member stars. Shaded elements represent stars which radial velocity differs by more than $3 \sigma$ from the cluster mean velocity.

Table 7. Cluster membership of program stars from the literature according to astrometric, photometric, and spectral type criteria, and ours based on spectrophotometric parallaxes and radial velocities. The last column gives our final membership status obtained by merging the results of the various criteria. $S 73=$ Sanders (1973), D02 $=$ Dias et al. (2002), $C 77=$ Crawford et al. (1977), J83 = Joshi et al. (1983), WOO= Wang \& $\mathrm{Hu}(2000)$.

\begin{tabular}{|c|c|c|c|c|c|c|c|c|}
\hline & \multicolumn{2}{|c|}{ astrom. } & \multicolumn{2}{|c|}{ photom. } & spctr & \multicolumn{2}{|c|}{ ours } & \\
\hline$\#^{-}$ & $\overline{S 73}$ & $\overline{\mathrm{D} 02}$ & C77 & $\overline{J 83}$ & $\frac{1}{\mathrm{~W} 00}$ & dist. & & \\
\hline 1 & $\mathrm{y}$ & $\mathrm{n}$ & $\mathrm{n}$ & $\mathrm{n}$ & $\mathrm{n}$ & & $\mathrm{n}$ & $\mathrm{N}$ \\
\hline 2 & $\mathrm{y}$ & $\mathrm{y}$ & $y$ & $y$ & $\mathrm{y}$ & y & $\mathrm{y}$ & $\mathrm{Y}$ \\
\hline 3 & $\mathrm{y}$ & $\mathrm{y}$ & $y$ & $\mathrm{y}$ & $\mathrm{y}$ & $\mathrm{y}$ & $\mathrm{y}$ & Y \\
\hline 4 & $\mathrm{y}$ & $\mathrm{y}$ & $\mathrm{y}$ & $\mathrm{y}$ & $\mathrm{y}$ & y & $\mathrm{y}$ & $\mathrm{Y}$ \\
\hline 5 & $\mathrm{y}$ & $\mathrm{y}$ & $\mathrm{y}$ & $\mathrm{y}$ & $\mathrm{y}$ & & $\mathrm{y}$ & $\mathrm{Y}$ \\
\hline 6 & $\mathrm{y}$ & $\mathrm{y}$ & $\mathrm{y}$ & $\mathrm{y}$ & $\mathrm{y}$ & & (y) & $\mathrm{Y}$ \\
\hline 7 & $\mathrm{y}$ & $\mathrm{y}$ & $\mathrm{n}$ & $\mathrm{y}$ & $\mathrm{y}$ & $\mathrm{y}$ & $\mathrm{y}$ & Y \\
\hline 8 & $\mathrm{y}$ & $\mathrm{y}$ & $\mathrm{y}$ & $\mathrm{y}$ & $\mathrm{y}$ & $\mathrm{y}$ & $\mathrm{y}$ & $\mathrm{Y}$ \\
\hline 9 & $\mathrm{n}$ & $\mathrm{n}$ & $y$ & & & $\mathrm{n}$ & $\mathrm{n}$ & $\mathrm{N}$ \\
\hline 10 & $y$ & $\mathrm{y}$ & $\mathrm{n}$ & $y$ & $\mathrm{y}$ & y & $\mathrm{y}$ & $\mathrm{Y}$ \\
\hline 11 & $\mathrm{n}$ & $\mathrm{n}$ & $\mathrm{n}$ & & & y & $\mathrm{n}$ & $\mathrm{N}$ \\
\hline 12 & $\mathrm{n}$ & $\mathrm{n}$ & $\mathrm{y}$ & & & $\mathrm{n}$ & $\mathrm{y}$ & $(\mathrm{N})$ \\
\hline 13 & $\mathrm{y}$ & $\mathrm{y}$ & $\mathrm{n}$ & $\mathrm{y}$ & $\mathrm{y}$ & y & $\mathrm{y}$ & $\mathrm{Y}$ \\
\hline 14 & $\mathrm{y}$ & $\mathrm{y}$ & $\mathrm{n}$ & $\mathrm{y}$ & $\mathrm{y}$ & $\mathrm{n}$ & $\mathrm{y}$ & $\mathrm{Y}$ \\
\hline 15 & $\mathrm{y}$ & $\mathrm{y}$ & & $\mathrm{n}$ & $\mathrm{y}$ & $\mathrm{y}$ & $\mathrm{y}$ & $\mathrm{Y}$ \\
\hline 16 & & $\mathrm{y}$ & & & & y & (y) & $\mathrm{Y}$ \\
\hline
\end{tabular}

\section{Internal kinematics of NGC 6913}

\subsection{A foreground cloud hiding part of the cluster}

As reviewed in the Introduction, all previous investigations of NGC 6913 agree on the large differential and total reddening affecting the cluster. Inspecting the Palomar charts it is evident how NGC 6913 lies close to a very thick interstellar cloud that seems to hide part of it.

Our and literature estimates about the cluster distance converge toward a $1.6 \mathrm{kpc}$ value. Studies of the interstellar extinction toward NGC 6913 (Crawford et al. 1977; Neckle \& Klare 1980) agree on a steep increase of the extinction at $1 \mathrm{kpc}$, about half the distance to the cluster, as if a single, major cloud is responsible for the majority of the extinction of NGC 6913.

In Fig. 6 we compare the same field centered on NGC 6913 as seen on Palomar POSS-II blue charts and by the IRAS satellite at $100 \mu \mathrm{m}$. The north-east quadrant is clearly deprived of stars in the optical image, while it is bright in the far infrared, a clear sign of thick dust absorbing in the optical and emitting in the IR. Star counts from USNO-A2.0 (stars detected in both blue and red POSS-I prints) and near-infrared 2MASS survey (stars detected both in $J$ as well as $H$ and $K$ bands) strongly support the argument of a strong foreground interstellar extinction crossing the field of NGC 6913 and increasing steeply toward the north-east quadrant.

We expect this foreground optically thick cloud to hide from view part of the cluster, even if the cluster center seems confidently identifiable with the grouping of the massive $\mathrm{O}$ and B stars seen in the optical.

\subsection{Cluster mass}

There are no published estimates of the NGC 6913 total mass. Two static approaches are considered in this subsection, a dynamical one is investigated in Sect. 3.4.

A lower limit to the cluster mass is obtained by adding the mass appropriate to the spectral type of known members (luminous mass). Taking Wang \& Hu (2000) spectral types of all S73 probable members classified by them and calibration into masses from Straižys \& Kuriliene (1981), we have

$M_{\mathrm{cl}} \simeq 700 M_{\odot}$.

S73 warned that a sizeable fraction of his members could be spurious. We assume here that they compensate for those fainter than the S73 completness magnitude, and therefore $700 M_{\odot}$ is taken as a fair indication of the total luminous mass of the cluster.

Assuming that the members of NGC 6913 distribute according to the Salpeter (1955) law $N(m)=C m^{-2.35}$ offers another possibility to estimate the cluster mass. It seems fair to assume that all $\mathrm{O}$ and $\mathrm{B}$ type cluster members have been detected and recognized as such in the Wang \& Hu (2000) spectral survey of NGC 6913. They are 31 in total, spanning the range between 6 and $67 M_{\odot}$. This allows to estimate the constant $C$

$31=C \int_{6}^{67} m^{-2.35} \mathrm{~d} m \quad \longrightarrow \quad C=489$

which provides an initial total stellar mass for the cluster amounting to

$M_{\mathrm{cl}}=489 \int_{0.08}^{120} N(m) m \mathrm{~d} m \simeq 3100 M_{\odot}$

distributed in about $10^{4}$ member stars. This is an upper limit to current cluster mass, because $(a)$ stars became unbound early in the cluster evolution when it started to lose gaseous mass blown away by the energetic winds of the first massive stars that formed, $(b)$ the relaxation mechanism leads to evaporation of the lighter members, and $(c)$ the mass function appears 


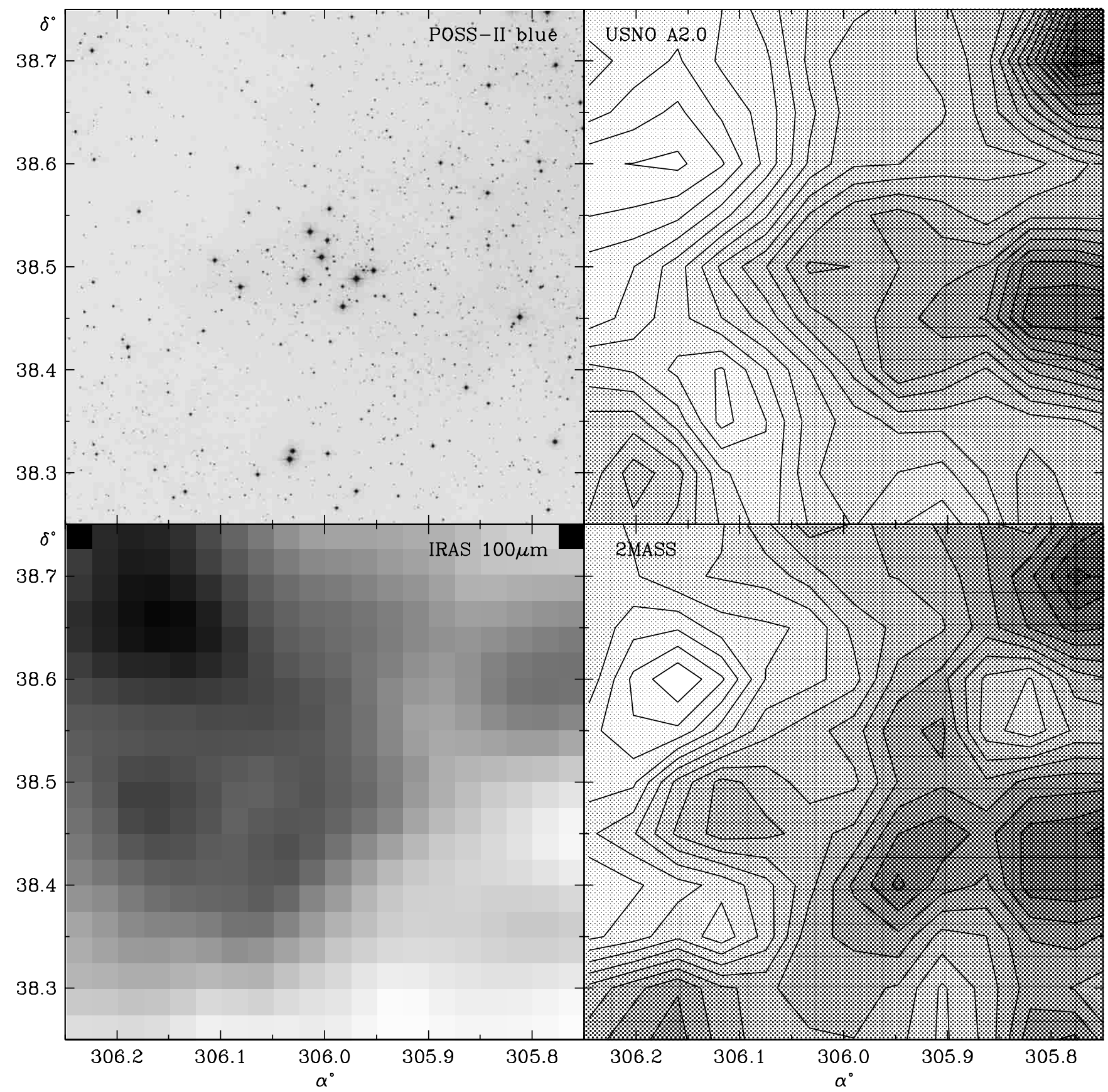

Fig. 6. Comparison of a $30^{\prime} \times 30^{\prime}$ field from POSS-II (top left) and IRAS $100 \mu \mathrm{m}$ wavelength image (bottom left) centered on NGC 6913 . Stellar surface density of the same stellar field is obtained from USNO-A2.0 optical catalog (top right) with minimum and maximum density of 0.5 and $4.7 \mathrm{stars} / \operatorname{arcmin}^{2}$ (clearest and darkest zone) respectively and grey levels in step of $0.25 \mathrm{stars} / \operatorname{arcmin}^{2}$, and from $2 \mathrm{MASS}$ infrared catalog (bottom right) with minimum and maximum density of 6.9 and $14.2 \mathrm{stars} / \mathrm{arcmin}^{2}$ (clearest and darkest zone) respectively and grey levels in step of 0.5 stars/arcmin ${ }^{2}$.

to flatten toward lower masses (cf. Briceño et al. 2002). The Salpeter and observed mass functions are compared in Fig. 7. In Sect. 3.5 and Fig. 8 we will show how there is possible evidence that the cluster is at least partially relaxed. Supposing that the associated evaporation of members affects stars fainter than the S73 completness magnitude, it is found that

$M_{\mathrm{cl}}=489 \int_{1.2}^{67} N(m) m \mathrm{~d} m \simeq 1000 M_{\odot}$ distributed in 350 members, comparable to the luminous mass in Eq. (1).

\subsection{Tidal and half-mass radii}

Cluster member venturing on orbits wider than the cluster tidal radius have a fair chance to become unbound due to the action 


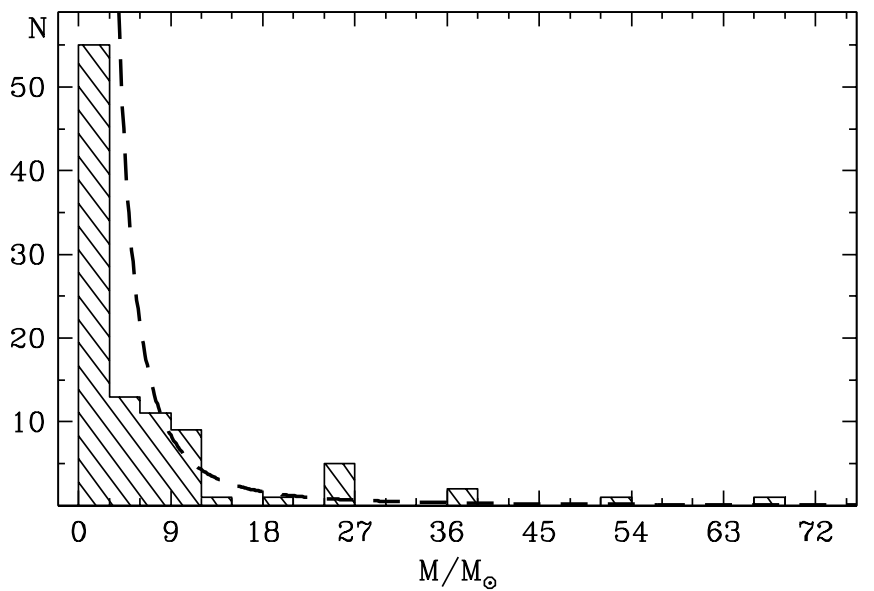

Fig. 7. Mass function of the cluster NGC 6913 as it results from the spectral classification of the member stars. The dashed line is a fit with a Salpeter law $N(m)=489 m^{-2.35}$ to O and B stars $\left(M \geq 6 M_{\odot}\right)$.

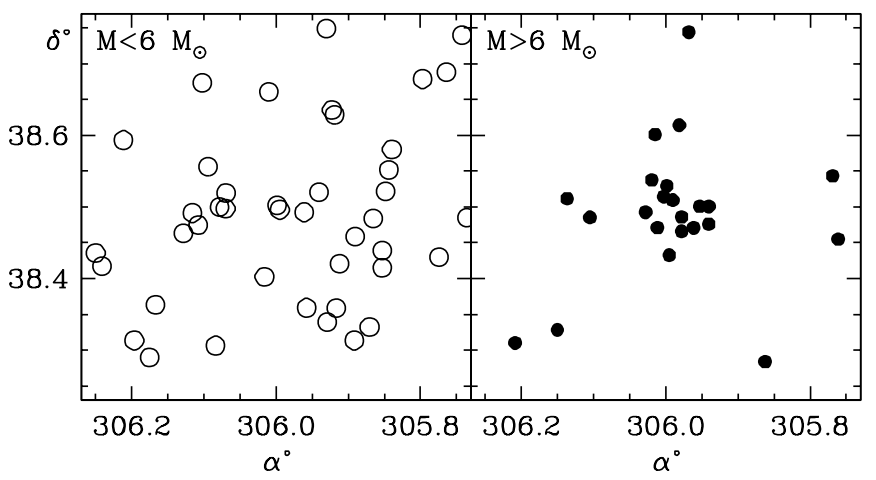

Fig. 8. Member stars of NGC 6913 with mass smaller (left) and larger (right) then $6 M_{\odot}$. Massive stars show a clear clustering while the lighter seem randomly distributed.

of the gravitational field of the Galaxy. The cluster tidal radius is defined as (cf. Binney \& Tremaine 1987):

$R_{\mathrm{t}}=R_{\mathrm{G}}\left(\frac{M_{\mathrm{cl}}}{3 M_{\mathrm{G}}}\right)^{\frac{1}{3}}$

where $R_{\mathrm{G}}, M_{\mathrm{G}}$ are the radius of the cluster galactic orbit and the galactic mass contained within $\left(R_{\mathrm{G}}=8.3 \mathrm{kpc}\right.$ and $M_{\mathrm{G}}=$ $\left.9.5 \times 10^{10} M_{\odot}\right)$. Inserting $M_{\mathrm{cl}}=700 M_{\odot}$ provides $R_{\mathrm{t}} \sim 10 \mathrm{pc}$, corresponding to $20^{\prime}$ at the estimated $1.6 \mathrm{kpc}$ cluster distance. On photographs, the cluster tidal radius is conventionally taken as the distance from the cluster center at which the stellar density drops to the field value, estimated by Lyngå (1987) to be $\sim 10^{\prime}$ for NGC 6913, which corresponds to $4.7 \mathrm{pc}$ at the adopted cluster distance $1.6 \mathrm{kpc}$. For $R_{\mathrm{t}}=4.7 \mathrm{pc}$ Eq. (5) would provide a much lighter cluster mass, $M_{\mathrm{cl}}=50 M_{\odot}$. Equation (5) describes the statistical relation of equilibrium between cluster and galactic potentials that can be realized only on time scales long enough for perturbation fluctuations to average. Given the very young age of NGC 6913 (corresponding to just 2\% of its galactic orbital period), the conditions supporting Eq. (5) can hardly be matched, and an $M_{\mathrm{cl}}$ estimated via Eq. (5) cannot be trusted. For the same reason, the $R_{\mathrm{t}}$ estimated from star counts has to be preferred to the value estimated using Eq. (5).
The half-mass radius $\left(R_{\mathrm{hm}}\right)$ is a useful quantity frequently used in $N$-body simulations. We have estimated it by measuring the radius that contains half of the $700 M_{\odot}$ luminous mass of Eq. (1), which turned out to be $8^{\prime}$, corresponding to $R_{\mathrm{hm}}=$ $3.7 \mathrm{pc}$ at a distance of $1.6 \mathrm{kpc}$. Following Binney \& Tremaine (1987) the virial radius can be expressed as

$R_{\mathrm{vir}}=\frac{R_{\mathrm{hm}}}{0.4} \simeq 9 \mathrm{pc}$.

\subsection{Internal velocity dispersion and dynamical mass}

The mean radial velocity of NGC 6913 members is

$R V_{\odot}^{\mathrm{cl}}=-16.9 \pm 0.6 \mathrm{~km} \mathrm{~s}^{-1}$

with an observed dispersion of $\sigma_{\mathrm{obs}}=2.0 \mathrm{~km} \mathrm{~s}^{-1}$. The average uncertainty of the radial velocity of individual members is $\sigma_{\text {instr }}=1.0 \mathrm{~km} \mathrm{~s}^{-1}$, which leads to an intrinsic radial velocity dispersion of NGC 6913 members amounting to

$\sigma_{\mathrm{rv}}=1.70 \mathrm{~km} \mathrm{~s}^{-1}$,

the three quantities being related as $\sigma_{\mathrm{rv}}=\sqrt{\sigma_{\mathrm{obs}}^{2}-\sigma_{\text {instr }}^{2}}$. Many of the physical quantities for NGC 6913 derived in the following critically depend upon this number. We are confident that our observations, reductions and measurements are stateof-the-art and involve so many high resolution observations of such a large number of stars over a so long period of time, that doing much better with 2 m-class telescopes is hardly feasible. On the other hand, the high competition to access larger instruments prevents them to be assigned on programs like the present one that require such a massive telescope usage (even if equipped with MOS devices). Undoubtly, observations of more member stars over longer period of times would be useful to better constrain the intrinsic radial velocity dispersion, but this does not appear as an easy task. Therefore, we believe that in absence of deeper/better efforts, our measurement of the intrinsic radial velocity dispersion of NGC 6913 is worth some dynamical considerations that we develop in the rest of this paper.

The virial theorem links the cluster mass $M$ within the radius $R$ to the velocity dispersion in the form

$\overline{v^{2}}=\frac{G M}{2 R_{\mathrm{vir}}}=3 \sigma_{\mathrm{rv}}^{2}$.

For a virial radius of $9 \mathrm{pc}$, the virial cluster mass becomes

$M_{\mathrm{tot}}=\frac{6 R_{\mathrm{vir}} \sigma_{\mathrm{rv}}^{2}}{G} \simeq 4 \times 10^{4} M_{\odot}$.

There is a large difference between luminous and virial total mass for the cluster (a factor of sixty, $7 \times 10^{2}$ vs. $4 \times 10^{4} M_{\odot}$ ). Could it be intra-cluster mass? Wang \& Hu (2000) have derived a differential reddening across the cluster amounting to $\Delta E_{B-V}=1.82 \mathrm{mag}$. Even supposing the effect is solely caused by intra-cluster extinction (from the above discussion the major contribution to differential reddening should actually come from the optically thick foreground dust cloud) the amount of dust required to produce it is of the order of $4 \times 10^{3} M_{\odot}$. This is derived assuming a simple spherical symmetry, constant density, a standard dust-to-gas ratio 
$\left(N(H)=5.8 \times 10^{21} E_{B-V}\right.$ atoms $\mathrm{cm}^{-2}$, Kilian 1992) and that a $\Delta E_{B-V}=1.82$ mag differential extinction is accumulated as result of the line of sight crossing the whole cluster. Even under these assumptions the corresponding amount of intracluster material is far from filling the gap between the luminous (i.e. Eq. (1)) and dynamical mass (i.e. Eq. (10)).

Possible explanations for such a discrepancy could be any combination of the following effects:

(i) the large and optically thick interstellar cloud discussed in Sect. 3.1 hides from view a significant portion of the cluster, causing an underestimate of the tidal radius and luminous mass from star counts. Deep infrared imaging at $K$ band and longer wavelengths could test this scenario;

(ii) the cluster is still relaxing and some of the stars considered as members are actually unbound, leaving the cluster at velocities just larger than the escape one, inflating the apparent dispersion of radial velocities. An example could be star \#6 which is a member according to both astrometric investigations and combined photometric+spectroscopic criteria, and lies projected close to cluster center sporting one of the earliest spectral types (B0 V). Its radial velocity is well determined as $-25.6( \pm 0.9) \mathrm{km} \mathrm{s}^{-1}$, which is however $8.7 \mathrm{~km} \mathrm{~s}^{-1}$ away from the cluster mean velocity of $-16.9( \pm 0.6) \mathrm{km} \mathrm{s}^{-1}$. The difference exceeds $4 \sigma_{\text {obs }}$. The escape velocity from NGC 6913 is

$v_{\mathrm{esc}}=\sqrt{12 \sigma_{\mathrm{rv}}^{2}}=5.9 \mathrm{~km} \mathrm{~s}^{-1}$

supporting the possibility that star \#6 is actually escaping. Kroupa et al. (2001) $N$-body simulations of young clusters confirm that the observed dispersion of radial velocities could be biased by unbound members;

(iii) some cluster members could be components of very wide binaries, which orbital motion amount to just a few $\mathrm{km} \mathrm{s}^{-1}$ and cannot be detected by observations spanning less than some decades;

(iv) a cluster angular momentum larger than zero, with the axis pointing away from the line of sight, could pass unnoticed given the restricted number of observed stars and still contribute a fraction of the observed radial velocity dispersion. A cluster rotation having a projected radial component of $\sim 1.5 \mathrm{~km} \mathrm{~s}^{-1}$ at half mass radius could be enough to bring the virial mass in agreement with the luminous mass.

\subsection{Evaporation of members}

Lighter cluster members seem to be evaporating from NGC 6913 as the comparison in Fig. 7 between the observed and Salpeter mass function supports, and as mass segregation in Fig. 8 suggests.

In fact, for $\mathrm{O}$ and $\mathrm{B}$ type members the mean mass is $\bar{m} \simeq$ $22 M_{\odot}$ and $\sigma_{\mathrm{rv}}=1.70 \mathrm{~km} \mathrm{~s}^{-1}$ being the radial velocity intrinsic dispersion, the mean kinetic energy of $\mathrm{O}$ and $\mathrm{B}$ type members is

$\overline{E_{\mathrm{c}}}=\frac{1}{2} \bar{m} v^{2}=\frac{3}{2} \bar{m} \sigma_{\mathrm{rv}}^{2}=1.9 \times 10^{45} \mathrm{erg}$.

If the cluster is relaxing toward energy equipartition, this is the mean kinetic energy to which its members are aiming, and there must be a value of the mass below which the velocity exceeds the escape value and the stars tend to evaporate. Such limiting mass $m_{\text {lim }}$ follows from

$\frac{1}{2} m_{\mathrm{lim}} v_{\mathrm{esc}}^{2}=\overline{E_{\mathrm{c}}}=1.9 \times 10^{45} \mathrm{erg} \longrightarrow m_{\mathrm{lim}}=5.5 M_{\odot}$.

However, this is actually an upper limit to the mass below which the members tend to leave the cluster due to evaporation. If the cluster core seems already relaxing in spite of the very young age, this could not be the case for the lower density outer regions of the cluster, with the relaxation being a process moving outward from the cluster center on timescales longer than the NGC 6913 age. Only devoted, deep photometric investigations can address what the turning mass in NGC 6913 is for which lighter members are already experiencing evaporation and what its radial dependence is.

\subsection{Crossing time, relaxing time, mass segregation}

Crossing time and relaxing time are theoretical quantities which play a relevant role in cluster dynamics. They are closely related to the mass of the cluster, its dynamical status and the number of members. The crossing time is related to virial radius and cluster mass by ( $v$ from Eq. (9))

$t_{\mathrm{cr}}=\frac{2 R_{\mathrm{vir}}}{v}=\sqrt{\frac{8 R_{\mathrm{vir}}^{3}}{G M_{\mathrm{cl}}}}$

and the relaxing time relates to the number of members, cluster mass and half mass radius as

$t_{\text {relax }}=\sqrt{\frac{R_{\mathrm{hm}}^{3}}{G M_{\mathrm{cl}}}} \frac{N}{8 \log (0.4 N)}$

(from Spitzer 1987, derived for the case of globular clusters with similar mass members).

In the case of the luminous mass, $M_{\mathrm{cl}} \sim 700 M_{\odot}$ and $N=92$ (cf. Sect. 2.4), it is $t_{\mathrm{cr}}=45 \mathrm{Myr}$ and $t_{\text {relax }}=30 \mathrm{Myr}$, significantly longer that the estimated cluster age ( $\sim 5 \mathrm{Myr})$. For the Salpeter mass $M_{\mathrm{cl}} \sim 3100 M_{\odot}$ and $N=10^{4}$ (cf. Eq. (3)), the crossing time reduces to $t_{\mathrm{cr}}=20 \mathrm{Myr}$ while the relaxation time goes up to $t_{\text {relax }}=650 \mathrm{Myr}$, both still larger than the cluster lifetime.

NGC 6913 appears relaxed, at least in its core (where the relaxation time is expected to be shorter given the higher mass density) as mass segregation in Fig. 8 shows. Note that concentration of massive stars toward the cluster center is observed in some clusters to be present since their birth and not as the result of purely dynamical evolution (i.e. the Orion trapezium system, see Hillenbrand \& Hartmann 1998) and that some $N$-body simulations (Portegies Zwart et al. 2001) show mass segregation to happen in clusters over ages shorter then the canonical $t_{\text {relax }}$. So, the apparent concentration of heavier members toward the center of NGC 6913 could also be due to mechanism other than dynamical relaxation.

\subsection{Binaries}

The dispersion of velocities in NGC 6913 is $\sigma=\sqrt{3} \sigma_{\mathrm{rv}}=$ $2.9 \mathrm{~km} \mathrm{~s}^{-1}$. Binaries with orbital velocities faster than this tend 
to survive close encounters, those orbiting slower risk ionization (Kroupa 2000). An orbital velocity of $2.9 \mathrm{~km} \mathrm{~s}^{-1}$ corresponds to an orbital period of $10^{6.3}$ days for a binary with a total mass of $5.5 M_{\odot}$, which would go clearly undetected in the course of a 6-yr long monitoring program like ours. Such a binary would have an angular separation of $0.4 \operatorname{arcsec}$ (within the detection threshold of current observational techniques from the ground) which would rise to 4.4 arcsec for members with a total mass of $67 M_{\odot}$ and decrease to 0.07 arcsec for members with a total mass of $1 M_{\odot}$.

All binaries detected in this investigation appear strongly bound, not ionizable by close encounters with other cluster members, and quite probably primitive (the short cluster age argue against a capture scenario). Their large eccentricities and non synchronous orbits indicate how far they still are from tidal circularization of the orbits and locking of the rotation and orbital periods which characterize the field binaries. Wider, more ionizable binaries are beyond the realm of spectroscopy, and would be profitably searched for by high spatial resolution imaging.

\section{Desiderata}

A deep and wide field photometric investigation of NGC 6913 and surrounding field would be a good starting point to better constraint the total mass, tidal radius and drop in the luminosity function of the cluster, and to address the large discrepancy between observed luminous mass, integrated IMF mass and the observed virial mass. At the cluster distance and reddening, $U B V R I$ photometry complete to $V=20$ will map all cluster members more massive than $0.8 M_{\odot}$, thus venturing well into the realm of masses that should be already evaporating from the cluster. Such a photometric investigation, which is highly encouraged, should extend over a radius of not less that $20^{\prime}$ from the cluster center and should include $J H K L$ bands to overcome the very strong differential extinction caused by the foreground interstellar cloud discussed in Sect. 3.1. Once members lighter than those here investigated will be firmly identified, a study of their radial velocity distribution would add constraints to the kinematical status and evolution scenario of NGC 6913.

Acknowledgements. We would like to thank R. Griffin for the provided software and P. M. Marrese for securing the four spectra obtained during 2003. CB has been finacially supported by ASI I-R-050/02 and I-R-117/01 grants.

\section{References}

Bertelli, G., Bressan, A., Chiosi, C., Fagotto, F., \& Nasi, E. 1994, A\&AS, 106, 275

Bessell, M. S. 2000, PASP, 112, 961

Binney, J., \& Tremaine, S. 1987, Galactic Dynamics (Princeton: University Press)

Briceño, C., Luhman, K. L., Hartmann, L., Stauffer, J. R., \& Kirkpatrick, J. D. 2002, ApJ, 580, 317

Crawford, D. L., Barnes, J. V., \& Hill, G. 1977, AJ, 82, 606

Dias, W. S., Lepine, J. R. D., \& Alessi, B. S. 2002, A\&A, 388, 168

FitzGerald, M. P. 1970, A\&A, 4, 234

Hillenbrand, L. A., \& Hartmann, L. W. 1998, AJ, 492, 540

Hoag, A. A., Johnson, H. L., Iriarte, B., et al. 1961, Publ. US. Nav. Obs., 17,347

Hron, J. 1987, A\&A, 176, 34

Johnson, H. L. 1962, in Nebulae and Insterstellar Matter, ed. B. M. Middlehurst \& L. H. Aller, Stars and Stellar Systems series (The University of Chicago Press)

Joshi, U. C., Sanwal, B. B., Sagar, R., et al. 1983, PASJ, 35, 405

Kazlauskas, A., \& Jasevicius, V. 1986, Bull. Vilnius Astron. Obs., 75, 18

Kilian, J. 1992, A\&A, 262, 171

Kroupa, P. 2000, in Massive Stellar Clusters, ed. A. Lancon \& C. Boily, ASP Conf. Ser., 211, 233

Kroupa, P., Aarseth, S., \& Hurley, J. 2001, MNRAS, 321, 699

Liu, T., Janes, K. A., \& Bania T. M. 1989, AJ, 98, 2, 626

Liu, T., Janes, K. A., \& Bania, T. M. 1991, AJ, 102(3), 1103

Lyngå, G. 1987, Catalogue of Open Cluster Data, 5th ed.

Massey, P., Johnson, K. E., Degioia-Eastwood, K., et al. 1995, ApJ, 454,151

Morgan, W. W., \& Harris, D. L. 1956, Vis. Astron., 2, 1124

Munari, U., \& Tomasella, L. 1999, A\&A, 343, 806

Neckel, Th., \& Klare, G. 1980, A\&AS, 42, 251

Peña, J. H., Peniche, R., Garcia Cole, A., et al. 2001, Odessa Astron. Pub., 14, 159

Portegies Zwart, S., McMillan, S. W., Hut, P., et al. 2001, MNRAS, 321, 199

Salpeter, E. E. 1955, ApJ, 121, 161

Sanders, W. L. 1971, A\&A, 14, 226

Sanders, W. L. 1973, A\&A, 9, 221

Spitzer, L. 1987, Dinamical evolution of Globular Cluster (Princeton: University Press)

Straižys, V. 1977, Multicolor Stellar Photometry (Vilnius: Mokslas Publishers)

Straižys, V., \& Kuriliene, G. 1981, Ap\&SS, 80, 353

Tifft, W. G. 1958, AJ, 63, 127

Wang, J. J., \& Hu, J. Y. 2000, A\&A, 356, 118

Yamashita, Y., Nariai, K., \& Norimoto, Y. 1977, An Atlas of Representative Stellar Spectra (The Univ. of Tokyo Press) 


\section{Online Material}


Table 2. Journal of observations. $D$ is the dispersions $(\AA /$ pix $)$ at $\mathrm{H} \alpha(0.19$ corresponding to unbinned spectra, 0.38 to $2 \times$ binned spectra), and $\Delta \lambda$ is the wavelength coverage. The last column gives the program stars observed in each given run (the table is only available in electronic form).

\begin{tabular}{|c|c|c|c|}
\hline date & $\mathrm{D}$ & $\Delta \lambda(\AA)$ & star \# \\
\hline 1996.06 .28 & 0.38 & $4100-6700$ & $1,2,4,5,6,7,10,12,14$ \\
\hline 1996.06.29 & 0.38 & $4100-6700$ & $3,8,9,11,13$ \\
\hline 1996.08 .01 & 0.38 & $4100-6700$ & $1,2,3,4,8,9$ \\
\hline 1996.08 .02 & 0.38 & $4100-6700$ & $5,6,10,11,12$ \\
\hline 1996.08 .03 & 0.38 & $4100-6700$ & $1,2,3,4,5,8,9$ \\
\hline 1996.08 .04 & 0.38 & $4100-6700$ & $6,10,11,12$ \\
\hline 1996.08.20 & 0.38 & $4100-6700$ & $3,6,7,10,11$ \\
\hline 1996.08.21 & 0.38 & $4100-6700$ & $1,2,4,5,8,9,12$ \\
\hline 1996.08 .28 & 0.38 & $4100-6700$ & $1,2,4,5,6,12$ \\
\hline 1996.08 .29 & 0.38 & $4100-6700$ & $3,7,8,9,10,11,13,14$ \\
\hline 1996.09.06 & 0.38 & $4100-6700$ & $1,2,3,4,5,6,7,8,9,12,14$ \\
\hline 1996.09.07 & 0.38 & $4100-6700$ & $5,10,13$ \\
\hline 1997.07 .25 & 0.38 & $4100-6700$ & 1,5 \\
\hline 1997.07 .26 & 0.38 & $4100-6700$ & $2,3,4,6,7,8,9,10,11,12$ \\
\hline 1997.07 .29 & 0.38 & $4100-6700$ & 13,14 \\
\hline 1997.07 .30 & 0.38 & $4100-6700$ & 11 \\
\hline 1997.08 .10 & 0.38 & $4100-6700$ & $2,3,4,7,8,9,10$ \\
\hline 1997.08 .11 & 0.38 & $4100-6700$ & $1,5,6,11$ \\
\hline 1997.08 .12 & 0.38 & $4100-6700$ & 12 \\
\hline 1997.08 .13 & 0.38 & $4100-6700$ & $1,4,7,11,12$ \\
\hline 1997.08 .14 & 0.38 & $4100-6700$ & $1,4,7,11,12$ \\
\hline 1997.08 .15 & 0.38 & $4100-6700$ & 11 \\
\hline 1998.09.09 & 0.38 & $4500-9500$ & $1,4,7,12$ \\
\hline 2000.09 .12 & 0.38 & $4500-9500$ & $2,3,6,8,13,14$ \\
\hline 2000.09 .13 & 0.38 & $4500-9500$ & $2,3,6,8,13,14$ \\
\hline 2000.09 .14 & 0.38 & $4500-9500$ & $2,3,6,8$ \\
\hline 2000.09 .16 & 0.38 & $4500-9500$ & $2,3,4,5,6,13,14$ \\
\hline 2000.09 .17 & 0.38 & $4500-9500$ & $1,2,6,7,14$ \\
\hline 2001.07 .27 & 0.19 & $4500-9500$ & $2,3,4,7,10$ \\
\hline 2001.07 .27 & 0.38 & $4500-9500$ & 6 \\
\hline 2001.07 .28 & 0.38 & $4500-9500$ & $7,10,15$ \\
\hline 2001.07 .28 & 0.19 & $4500-9500$ & $2,3,4,6$ \\
\hline 2001.07 .29 & 0.19 & $4500-9500$ & $2,3,4,6$ \\
\hline 2001.07 .29 & 0.38 & $4500-9500$ & 7,15 \\
\hline 2001.07 .29 & 0.19 & $4500-9500$ & $2,3,4,6$ \\
\hline 2001.07 .30 & 0.19 & $4500-9500$ & 1 \\
\hline 2001.09.09 & 0.38 & $4500-9500$ & 11,12 \\
\hline 2001.09 .09 & 0.19 & $4500-9500$ & 1 \\
\hline 2001.10.29 & 0.19 & $4500-9500$ & $1,2,3,4$ \\
\hline 2001.11.02 & 0.19 & $4500-9500$ & $1,4,6$ \\
\hline 2001.11.29 & 0.19 & $4500-9500$ & 2,3 \\
\hline 2001.11.30 & 0.19 & $4500-9500$ & $1,3,7,10$ \\
\hline 2001.12 .28 & 0.38 & $4500-9500$ & $3,7,15,16$ \\
\hline 2002.01 .02 & 0.38 & $4500-9500$ & 7,15 \\
\hline 2002.01 .03 & 0.38 & $4500-9500$ & 16 \\
\hline 2002.01 .04 & 0.38 & $4500-9500$ & $3,7,16$ \\
\hline 2002.01 .06 & 0.38 & $4500-9500$ & 15,16 \\
\hline 2003.05 .21 & 0.19 & $4500-9500$ & $1,5,16$ \\
\hline 2003.05 .22 & 0.19 & $4500-9500$ & 16 \\
\hline
\end{tabular}

